\title{
Estructuras y procesos de gobernanza en la política medioambiental: red de actores de la Comunidad Autónoma del País Vasco'
}

\author{
Alberto de la Peña Varona \\ Universidad del País Vasco \\ alberto.delapena@ehu.es
}

Resumen

En este trabajo se analiza la configuración de la red de actores público-privados en el ámbito de las políticas ambientales de la Comunidad Autónoma del País Vasco. El punto de partida de nuestro análisis será la entrada en vigor de la Ley 3/1998 de protección del medio ambiente ya que plantea un nuevo enfoque participativo que cambió en su día las estructuras de gobernanza. Para ello, hemos analizado el funcionamiento de diversos mecanismos participativos formales y no formales, partiendo de fuentes documentales y entrevistas en profundidad. Nuestras conclusiones nos llevan a destacar el valor del conocimiento como recurso clave que condiciona decisivamente la posición de cada uno de los actores en la red.

Palabras clave

Gobernanza, redes, política ambiental, grupos de interés, participación, País Vasco.

\section{Governance structures and processes in environmental policies: actors network in the Basque Country}

\begin{abstract}
In this paper we analyze the policy network of environmental policies in the Basque Country. Our starting point will be the approval of Law 3/1998 on environmental protection as it set up a new participative focus which changed governance structure. In order to do this, we have examined the functioning of formal and non-formal mechanisms of participation, through documental analysis and in-depth interviews. Our conclusions lead us to stress the value of knowledge as a condition for every actor to find their place in the network.
\end{abstract}

Keywords

Governance, networks, environmental policies, interest groups, participation, Basque Country.

1 Este trabajo ha sido posible gracias a la financiación del MICINN a través del Proyecto coordinado CSO2009-14381C03-01 y del Grupo de investigación consolidado del Gobierno Vasco IT-323-07. 


\section{INTRODUCCIÓN}

El concepto de redes de políticas públicas (policy networks) se ha revelado como una excelente herramienta analítica a la hora de examinar el proceso de diseño e implantación de las políticas públicas, partiendo de la base de analizar a éstas más como resultado de una interacción entre varios actores que como producto de una decisión. Existen diferentes escuelas o formas de entender las redes (Börzel, 1997); cabe distinguir, por un lado, la corriente anglosajona que entiende las policy networks como un aspecto más de la intermediación de intereses y, por el otro, una perspectiva europeo continental más preocupada en relacionarlas con la gobernanza. En este trabajo nos situaremos más próximos quizá a este segundo enfoque, analizando la red de la política ambiental como una consecuencia necesaria de un determinado sistema de gobernanza en estrecha relación con el contexto institucional en el que aparece (Klijn, 1998).

En general, el concepto de redes nos habla de una pluralidad de actores que establecen entre sí vínculos de cooperación más o menos estables en un contexto de mutua interdependencia (Arenilla, 2011: 34; Börzel, 1997: 1; Chaqués, 2005: 36); como es sabido, este conjunto de actores tiene naturaleza público-privada, reservándose generalmente para el Estado cierto papel de catalizador (Natera, 2004) que tenderá a buscar en las redes tanto legitimidad como resolución efectiva de problemas políticos en un contexto de creciente complejidad. En definitiva, la configuración de redes de políticas públicas no es sino la necesaria consecuencia de la búsqueda por parte del Estado, una vez reconocidas sus propias limitaciones, de colaboradores activos que aporten know-how y consenso a las decisiones públicas (Cerrillo, 2005). Partiendo de esta base, pretendemos aquí ver cómo se configura esa red sectorial en el caso concreto de la política ambiental, entendiendo ésta de una manera pragmática -una de las posibilidades que reconoce Aguilar (1997: 25; 1998: 249) cuando trata esta cuestión-, es decir, la que efectivamente desarrolla el departamento de medio ambiente en una estructura de gobierno determinada y que generalmente se descompone en cuatro grandes políticas sectoriales: agua, residuos, contaminación atmosférica y conservación de espacios naturales. Nos ceñiremos para ello al caso de la Comunidad Autónoma del País Vasco (CAPV).

Creemos que en nuestro objeto de estudio concurren una serie de factores que justifican un cierto interés a los ojos del analista de políticas públicas. En primer lugar, la política medioambiental representa un campo relativamente novedoso con respecto a otras políticas sectoriales, lo que se ve reflejado en un relativamente escaso número de trabajos que la analizan desde una perspectiva politológica. El que las políticas ambientales se hayan desarrollado de la forma que lo han hecho en los últimos años, unido a que la sostenibilidad -y dentro de ella, especialmente la ambiental- venga siendo reconocida como uno de los retos sistémicos más apremiantes, justifica, a nuestro modo de ver, que desde la Ciencia Política se vaya cubriendo ese vacío y vayamos teniendo un conocimiento más preciso de este campo de actuación

126 pública, a lo que, modestamente, pretende contribuir este trabajo. En segundo lugar, 
entendemos que un estudio de estas características en España por fuerza habría de remitirse al ámbito autonómico; tanto desde la perspectiva jurídica (Arluzea, 2006) como la politológica (Aguilar, 1999), se destaca que el reparto constitucional de competencias ambientales otorga un papel protagonista a las CCAA en el plano ejecuti$\mathrm{vo}^{2}$. Por ello, entendemos que será a nivel de estas donde encontraremos más claramente configuradas las redes de las políticas ambientales. En tercer lugar, los pronunciamientos institucionales desde la Cumbre de Río, en 1992, hasta el último Programa de Acción Medioambiental Europeo coinciden en señalar la participación como uno de los puntales de las políticas ambientales y de sostenibilidad, como así se ha evidenciado, por ejemplo, en las Agendas Locales 21. En ese sentido, creemos que cobra un interés especial contrastar cómo casan los principios participativos con los procesos reales de diseño e implantación de políticas.

Concretando más el objetivo, nuestra atención se centrará en el periodo que va desde 1998 hasta la actualidad, ya que, como justificaremos en el siguiente epígrafe, entendemos que durante esa época se da un cambio radical en el enfoque y contenidos sustantivos de la política ambiental vasca, lo que acaba configurando una nueva red de actores. En este sentido, centraremos nuestra atención fundamentalmente en las relaciones entre actores públicos -básicamente el departamento del gobierno autonómico con competencias en medio ambiente, es decir, la autoridad ambiental- y los grupos de presión representativos tanto de intereses públicos como privados, dejando a un lado por razones de espacio, otro componente del análisis de redes como es la cuestión multinivel. Por tanto, abordaremos en los siguientes apartados tanto el funcionamiento más formal de la red, principalmente a través de cauces participativos institucionalizados, como las dinámicas menos regladas, no por ello menos importantes. De esta forma, pretendemos fundamentar las conclusiones recogidas en el último punto. Para desarrollar nuestro análisis, atenderemos tanto a fuentes documentales institucionales como a la información provista por actores procedentes del mundo político, asociativo y empresarial. Este último caudal de datos proviene de las entrevistas en profundidad realizadas para un proyecto de investigación financiado por el Gobierno Vasco a través de la Cátedra UNESCO${ }^{3}$, y entendemos que complementa la información documental -el discurso de las instituciones- con interpretaciones más subjetivizadas, pero a la vez más matizadas y ponderadas, de cómo se diseñan e implementan las políticas ambientales.

2 Desde otra óptica, el nivel autonómico también parece ser percibido como especialmente relevante por los actores presentes en el proceso de implantación de políticas medioambientales en Cataluña (Morata y Hanf, 2000).

3 Las principales conclusiones del Proyecto UNESCO 06/14, “La Política Ambiental del Gobierno Vasco", se recogen en De la Peña et al., (2012), tratándose la política ambiental de una manera más genérica en cuanto a su evolución sustantiva, las relaciones intergubernamentales y la gobernanza. Para la realización del trabajo de campo de este proyecto se realizaron un total de 31 entrevistas a once altos cargos del Gobierno Vasco que desempeñaron su función en la consejería de medio ambiente en diferentes legislaturas, dos altos cargos de otras administraciones autonómicas, siete activistas de organizaciones ambientales y sindicales, cuatro representantes del mundo empresarial, seis del ámbito académico y uno de los medios de comunicación. 


\section{MECANISMOS FORMALES DE PARTICIPACIÓN Y GOBERNANZA}

\section{El influjo europeo de la política ambiental vasca}

Si hubiésemos de resumir la línea evolutiva de la política ambiental en España durante las últimas décadas, habríamos de incidir necesariamente en la idea de la influencia de las políticas europeas (Aguilar, 1997, 2003; Arias y Valencia, 2004; Navarro, 1999). El caso de la CAPV no va a ser contrario a esta pauta, si bien ello no equivale a sostener que la inclusión de las cuestiones ambientales en la agenda pública fuese producto exclusivamente del ingreso en la Comunidad Económica Europea; en realidad, determinadas actuaciones públicas en materia medioambiental habían venido dándose en Euskadi desde la década de los setenta, motivadas en un principio por el problema de la contaminación atmosférica y del agua (De la Peña y Barcena, 2012). Sin embargo, queda fuera de toda duda la influencia que a partir de 1986 habría de tener la integración en el espacio europeo, no solo en el aspecto sustantivo de las políticas ambientales, sino también en su enfoque.

Un punto de inflexión claro en la evolución de estas políticas podemos situarlo en 1998. En esa fecha se elabora la Ley 3/1998, de 27 de febrero, general de protección del medio ambiente del País Vasco (Boletín Oficial del País Vasco, 27 de marzo de 1998), donde se integran explícitamente los conceptos de Desarrollo Sostenible y participación, como venían siendo recogidos en los Planes de Acción Medioambiental europeos (Aguilar, 2003; Font, 2000). El influjo europeo de la norma viene explicitado en su propia exposición de motivos y se transmite, sobre todo, al abrir una nueva fase que podríamos definir como el nacimiento de la planificación estratégica en la política ambiental, puesto que como mandato de la Ley 3/1998 se elabora la Estrategia Ambiental Vasca de Desarrollo Sostenible 2002-2020 (EAVDS) y, en desarrollo de ésta, los sucesivos programas marco ambientales (PMA)4. A partir de este momento, las políticas ambientales tratarán de ser elaboradas mediante una participación lo más amplia posible, por lo que se crea el Consejo Asesor de Medio Ambiente (CAMA), se revaloriza el papel de otros consejos consultivos que ya existían y se recurre a procesos participativos ad-hoc para la elaboración de la planificación. Es esa fase iniciada entonces la que hemos elegido para examinar la configuración de la red de actores.

\section{EI CAMA: integrando a los actores (2002-2011)}

La Ley 3/1998, en sus artículos 12 al 15, crea el CAMA para dar cauce formal a la participación de todos los actores públicos y privados con interés en las políticas medioambientales a nivel de la CAPV55. Desde la primera línea se establece claramente

4 El I Programa Marco Ambiental tendrá vigencia para el periodo 2002-2006, el segundo para 2007-2010 y el tercero, recientemente aprobado por el Gobierno Vasco, para el periodo 2011-2014.

5 Aparte del CAMA, en la CAPV existen otros tres consejos sectoriales de participación en materia de medio ambiente a los que podríamos hacer extensivas buena parte de las conclusiones relativas al 
la función consultiva de este órgano que se adscribe a la autoridad ambiental del gobierno autonómico, y cuya composición y funcionamiento queda regulado por el Decreto 199/2000, de 10 de octubre, por el que se regulan la composición y funcionamiento del Consejo Asesor de Medio Ambiente de la Comunidad Autónoma del País Vasco (Boletín Oficial del País Vasco, 26 de octubre de 2000). Según la normativa, el consejo debería reunirse un mínimo de tres veces anuales y siempre que lo requiera el desempeño de sus funciones (art. 8) que pasan principalmente por asesorar en materia de política ambiental, emitir informes y propuestas a iniciativa propia o a petición de las administraciones y recomendar medidas que integren la variable ambiental en otras políticas sectoriales, así como impulsar la participación en esta política sectorial (art. 13 de la Ley 3/1998). En ese sentido, estimamos que el CAMA delimita un escenario en el que aparecen incluidos los principales actores de la red ambiental, si bien deberemos cotejar sus aspectos formales con las dinámicas de funcionamiento que se derivan de las apreciaciones de sus participantes.

Su composición cuenta con un total de treinta y un miembros, entre los cuales encontramos personas procedentes de los departamentos que gestionan el medio ambiente en los ejecutivos autonómico y forales ${ }^{6}$, representantes de otros departamentos del gobierno vasco, de la Asociación de Municipios Vascos (EUDEL) y portavoces de diferentes grupos y asociaciones con vinculación a la política ambiental; también centros tecnológicos y un experto de reconocido prestigio designado por el propio Gobierno Vasco. Si analizamos la composición del CAMA según la procedencia de sus miembros, vemos que quince del total de treinta y uno son cargos públicos ocho del Gobierno Vasco, incluyendo el presidente, tres de las Diputaciones, tres de las administraciones locales y uno del Parlamento Vasco-, doce corresponden a diferentes grupos de interés público y privado y cuatro al ámbito de la investigación -procedentes de centros tecnológicos, de investigación, la Universidad del País Vasco y un experto, definido como tal por la normativa y habitualmente también miembro de la Universidad. Ello queda ilustrado en el Gráfico 1.

Lo cierto es que de esta composición se puede concluir el papel absolutamente determinante de las instituciones en el supuesto de que las votaciones fueran necesarias para definir una postura colegiada. Normalmente, las cuestiones debatidas en el CAMA, ya sea en forma de textos normativos, planes o programas de actuación, vienen respaldadas por todos o la inmensa mayoría de los representantes de las diferentes administraciones. La explicación resulta sencilla: por una parte, a nivel de gobierno autonómico y por la propia lógica de funcionamiento del gabinete, las posibles disensiones se tienden a ventilar en fases previas a la presentación del proyecto ante el CAMA; por otra parte, y a nivel de relaciones interadministrativas, se supone que

CAMA en torno a su composición y dinámica de funcionamiento: Consejo Asesor de Política Territorial, Naturzaintza y Consejo Asesor del Agua (De la Peña et al., 2012). No obstante, hemos seleccionado el CAMA para nuestro análisis por la centralidad que le otorga la propia Ley 3/1998.

6 La normativa establece que serán miembros del CAMA, un representante de cada una de las Diputaciones Forales sin especificar la procedencia departamental. La práctica ha sido que acudan los diputados con responsabilidad en medio ambiente. 
otros ámbitos, como la Comisión Ambiental del País Vasco7, deberían resolver previamente las diferencias entre ejecutivos y cimentar una postura común ante una determinada política. Así, lo más habitual es que no se reproduzcan diferencias entre los representantes institucionales y que las líneas de actuación planteadas por el Gobierno Vasco tengan un apoyo garantizado en forma de votos, dado que es prácticamente imposible que haya unidad de voto en la parte asociativa del CAMA por la heterogeneidad de los intereses representados. Consecuentemente, la hipótesis más previsible es que las iniciativas de las instituciones, por el propio contenido de sus propuestas, encuentren siempre el apoyo de una parte de las organizaciones -ya sea en la sección empresarial, sindical o ecologista- y que sea la otra parte, los grupos opositores, los que capitalicen el CAMA para formular el discurso alternativo.

\section{GRÁFICO 1}

\section{Composición del CAMA según procedencia de sus miembros}

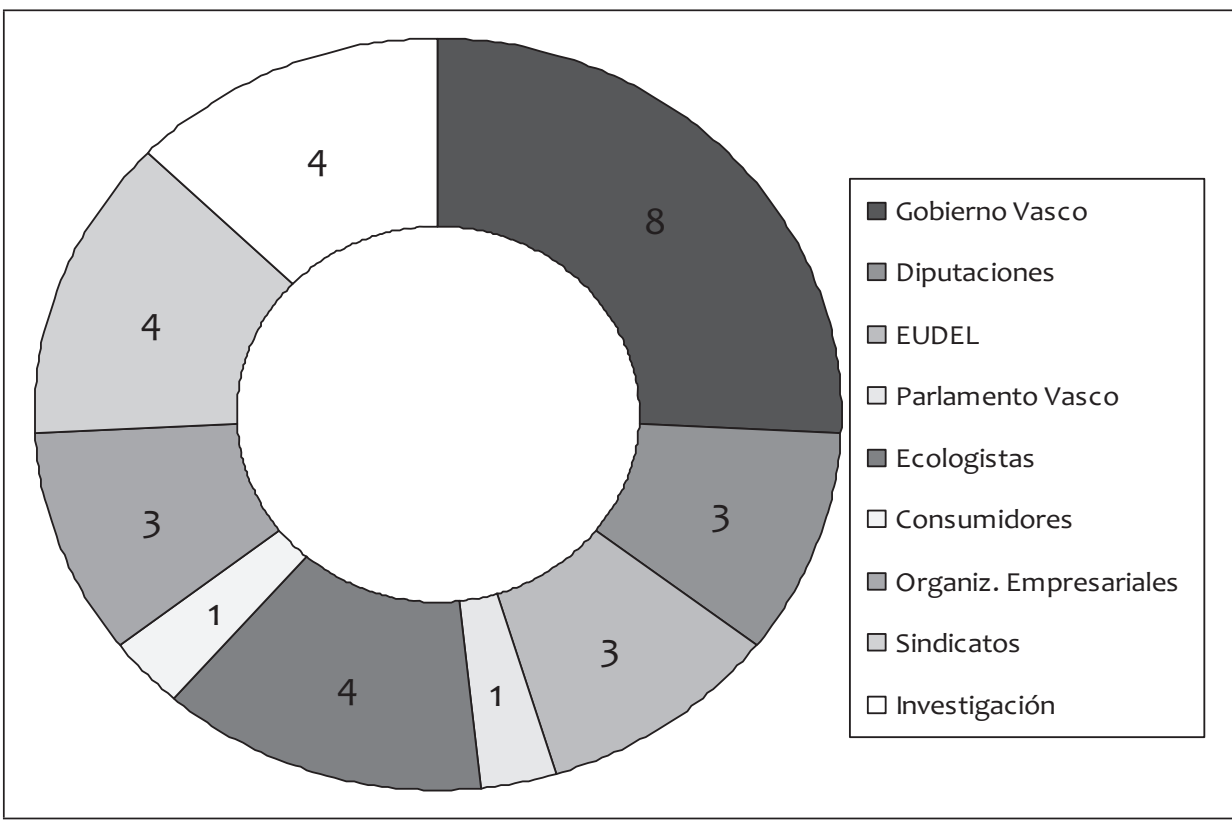

Fuente: Elaboración propia a partir del Decreto 199/2000 (art. 3)

Tal parece haber sido la tónica de funcionamiento del CAMA, si atendemos a los testimonios de las personas que hemos entrevistado y al contenido de las actas de sus reuniones. La nota predominante habría sido cierta dialéctica de gobierno-oposi-

7 Ya en el mismo texto de la Ley 3/1998 se puede apreciar cómo el órgano de coordinación interadministrativa -la Comisión Ambiental del País Vasco- precede al CAMA en la actuación sobre las políticas ambientales. Según el art. 9, corresponde a la Comisión, por ejemplo, informar el Programa Marco Ambiental o la elaboración de normas en la fase de anteproyecto. Como vemos, el diseño prevé que el CAMA actúe sobre esos mismos outputs, pero en una fase posterior. 
ción protagonizada, por un lado, por la administración ambiental autonómica y, por otro lado, por una especie de alianza entre asociaciones ecologistas y sindicales. De esta manera, no sorprende que los entrevistados pertenecientes a organizaciones ecologistas y sindicales mantengan una visión sumamente crítica con respecto al CAMA, definiéndolo como un escenario necesario pero, a la vez, notablemente limitado en sus funciones:

“A nivel global está el CAMA... con un funcionamiento al que nosotros hemos dirigido bastantes críticas desde su surgimiento; la propia composición, que es una composición claramente escorada hacia lo institucional. Pero, bueno, hemos participado junto con otros grupos ecologistas y es cierto instrumento de control y seguimiento, pero sigue siendo también muy limitado... No voy a decir que sea un organismo florero porque sirve por lo menos para tener información y cierta capacidad de crítica de las políticas medioambientales, pero creo que la eficacia real es escasa". (Entrevista № 1 . Activista Ekologistak Martxan).

"Al final, desde ese punto de vista, si la participación se reduce al CAMA, pues yo sinceramente alabo el gusto al compañero que va allí a sermonear. Pero, para mí es una pérdida de tiempo. Con la gran presencia institucional, ir allí a castigar el oído a quien no vas a convencer, y además sin ninguna proyección social, es una pérdida de tiempo". (Entrevista N²3. Miembro del Sindicato Comisiones Obreras, CCOO).

Como ya se puede apreciar en el anterior testimonio, se opone un discurso en el que a menudo aparece un componente de frustración por la escasa influencia de los debates; de hecho, frecuentemente se formula explícitamente la tesis de que el impacto real sobre las políticas se desarrolla en fases más tempranas, probablemente a través de mecanismos menos formalizados y por parte de grupos empresariales. Lo explica claramente otro de nuestros informantes:

"En las reuniones del CAMA... habitualmente es el gobierno que presenta una medida, una ley, un plan que quiere sacar adelante. Luego se hace una ronda, los representantes que estamos allí de sindicatos, de organizaciones ecologistas (cinco o seis) damos nuestra opinión, en la mayoría de los casos crítica, y el resto de las personas que allí acuden (gente en representación de las Diputaciones, alguna parlamentaria, gente de la patronal, etc.) ... mayormente esa gente guarda silencio. A lo más, alguien del departamento debate con nosotros y ya está. Esa suele ser, más o menos, la tónica general. Allí acuden más de una decena de personas que no tienen por costumbre dar su opinión y entonces el debate es muy pobre. Y luego, pues tampoco tenemos constancia que de lo que allí sale se lleve a ningún lado, o tenga alguna relevancia, o sea tenido en cuenta. Luego, ciñéndome a lo que me has preguntado qué consecuencias tiene, por ejemplo, las opiniones de la parte empresarial... Pues, por ejemplo, cuando nosotros estudiamos el Plan Energético del Gobierno vemos que tienen muchísima influencia. A veces hasta podemos dudar si el Plan lo está haciendo Iberdrola o lo está haciendo un Gobierno en el que está Ezker Batua, EA, etc.". (Entrevista Nº22. Miembro de LAB y Ekologistak Martxan).

Esta visión es hasta cierto punto compartida por parte de otros miembros del CAMA que representan intereses de naturaleza diferente a la de ecologistas y sindi- 
calistas. Desde el punto de vista de las organizaciones empresariales, uno de sus representantes aprecia también una cierta inactividad y escasa funcionalidad del CAMA, si bien -como es el caso del siguiente entrevistado- asimilándolo al funcionamiento de otros consejos ambientales:

“(... ) yo creo que tiene un funcionamiento muy mortecino, se reúne muy pocas veces, cuando se reúne es más un foro de ecologistas, y hace muy poco. Y todos los consejos asesores de medio ambiente tienen el mismo problema, que funcionan muy poco.

Hay bastantes (ecologistas), y es lógico porque tiene que haber, pero al final no se ha conseguido que no se convierta en un foro para que ecologistas a los que se hace poco caso, en su opinión, aprovechen para manifestar una serie de cosas... y allí hay una reunión de varias horas en la que no se habla de otras cosas. ¿El resultado al final? Pues muy poco. $Y$ en el consejo español pasa lo mismo... Yo creo que es bueno que haya un foro que es obligatorio, pero el efecto real es prácticamente cero". (Entrevista $\mathrm{N}^{\circ} 25$. Miembro de ACLIMA, Clúster medio ambiental de empresas de la (APV).

Uno de los aspectos más llamativos de esta interpretación es que el entrevistado defina al CAMA como un foro "de ecologistas", para enfatizar el protagonismo de éstos en las reuniones y debates. Ello no hace sino corroborar el anterior testimonio que daba cuenta de una tónica predominante de diálogo entre gobierno y asociaciones ecologistas y sindicales. El resto de miembros opta por un papel menos relevante. En unos casos porque su papel lo ha ejercido con anterioridad, por ejemplo, las instituciones no autonómicas en la Comisión Ambiental o, como dicen ecologistas y sindicalistas, las organizaciones empresariales a través de contactos informales. En otros casos porque no se aprecia la relevancia de la institución y los debates se orientan hacia aspectos demasiado cercanos a la filosofía política sin responder a los problemas planteados, de índole mucho más práctica.

Desde el Gobierno Vasco todo esto se analiza aludiendo a las propias limitaciones funcionales del CAMA, si bien se enfatiza en la apertura que ha significado la creación de esta institución en la que se han presentado las iniciativas más relevantes de la política ambiental, se aduce como explicación la organización del gobierno que atribuye competencias a otros departamentos de políticas de alta incidencia ambiental como es el caso de las infraestructuras:

"Yo creo que la información relevante de las cuestiones de medio ambiente ha pasado por el CAMA. Al CAMA hemos llevado al consejero de transportes o la consejera de Industria. Aun así no ha sido suficiente, quiero decir que han ido allí, han informado, pero, claro, el CAMA por su formato no permite que se abra un debate como un tema, como el TAV exige... Entonces nosotros hemos hecho las veces de facilitador, pero es que nosotros no somos competentes en infraestructuras y no podemos convertir el CAMA en lo que, por otra parte, sólo una parte del CAMA quería porque a otra parte no le interesaba". (Entrevista $N^{\circ} 20$. Ex alto Cargo de 132 Medioambiente del Gobierno Vasco). 
En este sentido, el CAMA ha supuesto un paso adelante en el rol que habitualmente se atribuye a las instituciones públicas en el paradigma de la gobernanza, el papel de facilitador y de legitimador, pero no ha podido entrar en competencias ajenas al medio ambiente por la propia organización funcional del ejecutivo autonómico. Parece haber una visión compartida acerca de su limitado impacto -condicionado por las características formales del mecanismo participativo- y de la dinámica de sus reuniones en las que se escenifica una continua dialéctica entre ejecutivo y asociaciones sindicales y ecologistas.

\section{Iniciativas participativas en planes y programas: el caso del proceso de participación en la elaboración del II PMA.}

Dejando a un lado los foros y consejos consultivos, otra manera de articular la inclusión de grupos de interés en la elaboración de las políticas ambientales consiste en la apertura de procesos participativos determinados para la preparación de planes y programas específicos. En concreto, analizaremos el proceso de elaboración del II Programa Marco Ambiental 2007-2010 que fue elaborado sobre un amplio proceso participativo con cierto carácter innovador y que se compone de diferentes herramientas. Así nos lo cuenta uno de sus organizadores:

“(... ) el (proceso participativo) del Programa Marco, hasta donde yo sé, ha sido una de las dinámicas más sofisticadas con las limitaciones que tenía...”. (Entrevista № 13. Consultor de Medio Ambiente) ${ }^{8}$.

Como lo define su documento base, el esquema del proceso participativo abarca dinámicas orientadas tanto a actores y grupos de la sociedad civil, como a otros agentes institucionales. En este sentido, examinaremos las tres dinámicas participativas utilizadas para la elaboración del II PMA: los denominados Desayunos por la Sostenibilidad, el Consejo Consultivo y los foros social y empresarial.

\section{a) Los Desayunos por la Sostenibilidad}

Tal y como se presentan, los Desayunos por la Sostenibilidad son una dinámica participativa diseñada para ofrecer un diagnóstico del peso relativo del vector medioambiental en la escala de valores socialmente predominante, y recabar una reflexión sobre el papel de ciertos factores clave para la sostenibilidad como la energía, la movilidad o el sistema de mercado en general. Se desarrollaron en dos jornadas, una de ellas en Bilbao, en marzo de 2006, y otra en San Sebastián, dos semanas más tarde. Fueron, por tanto, dos reuniones puntuales realizadas un año antes de la publicación final del II PMA, con formato de foro en las que se pretende pulsar la

8 A esa importancia del PMA, y de su proceso participativo, se suma una ventaja metodológica y es que todo ese proceso viene descrito con cierto grado de detalle. En Anexo 2 (Gobierno Vasco, 2006). 
opinión de determinados agentes económicos, académicos y sociales para enriquecer una labor de diagnóstico que se estima substancial. Entre la lista de invitados a participar se encuentran importantes empresas (Iberdrola o Petronor), clústers empresariales (ACLIMA o Clúster de la Energía), centros tecnológicos (IK-4 o Labein), organizaciones ecologistas (Greenpeace), cámaras de comercio, representantes del mundo universitario, etc.; si bien, el elemento empresarial pesa sobre todos los demás, según se aprecia en el Gráfico 2.

\section{GRÁFICO 2}

Proceso participativo en la elaboración del II PMA (Iniciativa "Desayunos por la Sostenibilidad")

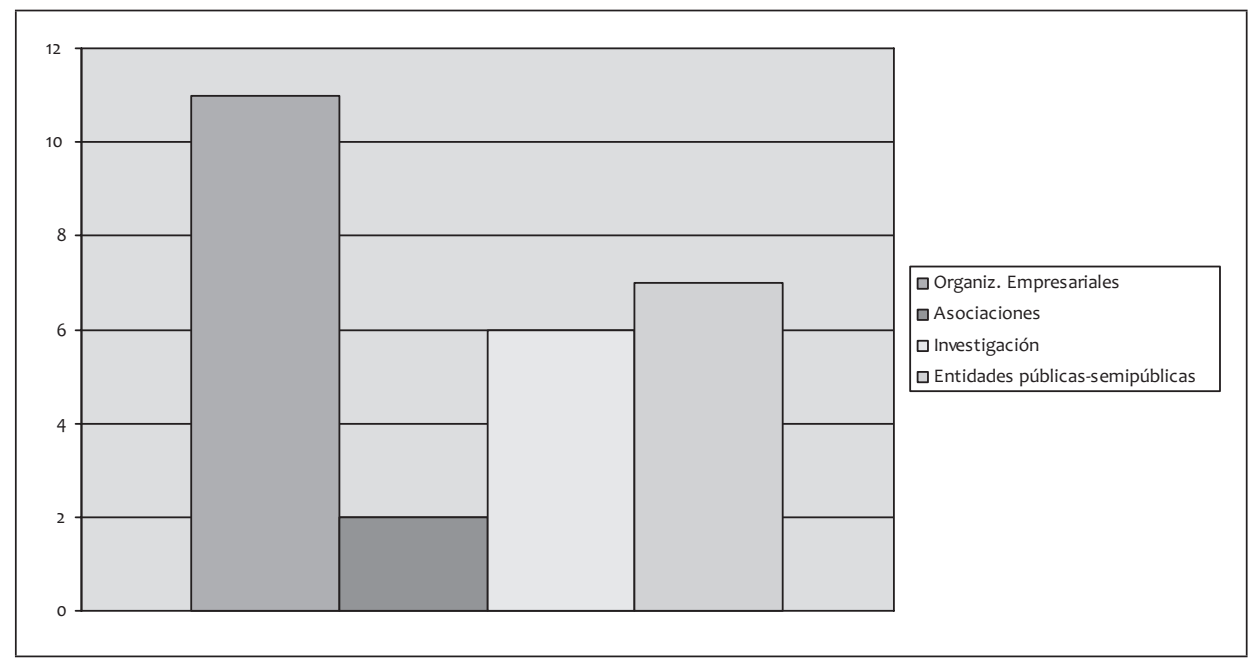

Fuente: Elaboración propia a partir de Gobierno Vasco (2006).

De esta dinámica participativa fueron extraídas una serie de conclusiones que se supone orientaron a la autoridad ambiental de la CAPV sobre el carácter que se debe imprimir al II PMA; en este sentido, se reafirma la validez de los cinco objetivos estratégicos definidos en la EAVDS, se recalca la importancia de un enfoque sistémico por la propia naturaleza de la política ambiental, se insiste en aspectos cruciales de ésta como el problema del cambio climático, etc. (Gobierno Vasco, 2006: 76).

\section{b) El Consejo consultivo}

A través del Consejo consultivo se trataba de complementar el diagnóstico así como avanzar en algunas prioridades de los objetivos del II PMA. La técnica empleada en esta dinámica participativa consistía en la realización de entrevistas en profundidad, donde la persona consultada exponía sus puntos de vista sobre la base de un

134 cuestionario previamente definido. Como las otras dinámicas del II PMA, la organiza- 
ción del Consejo corrió a cuenta de una consultoría especializada en estudios de sostenibilidad que organizó las sesiones y presentó una lista de participantes. Al respecto:

“(... ) los criterios de selección se hicieron con un proceso compartido entre nosotros que hacíamos de consultores y el cliente que era el Gobierno Vasco. Nosotros hacíamos las propuestas en base a unos criterios, y ellos te van diciendo OK o no... Pero normalmente la iniciativa es del equipo consultor que ya tiene una idea de lo que quiere hacer, y luego el Departamento te va filtrando. Es así cómo funciona...". (Entrevista No 13. Consultor de Medio Ambiente).

En esta parte del proceso, por su propio diseño, los participantes tenían mayor tiempo y oportunidad para exponer un discurso propio que reflejase sus intereses y puntos de vista, funcionando además de manera dialéctica con la administración, no como un órgano colectivo. Es importante tener esto en cuenta ya que esta profundidad de discurso no va a ser hallada en las otras dinámicas, donde el formato de foro diluye, en mayor medida, los intereses en una pluralidad de opiniones.

Los participantes en el Consejo consultivo fueron un total de ocho personas destacadas en el ámbito del medio ambiente por su trayectoria profesional. De estas ocho personas, cuatro son catedráticos de la Universidad del País Vasco, especializados en temas ambientales desde diferentes aproximaciones disciplinares; dos son representantes de distintas administraciones públicas (en concreto de la Diputación Foral de Bizkaia y de la Comunidad Autónoma de Baleares), uno es representante de una asociación como la Corte Internacional de Arbitraje y Conciliación Ambiental también académico- y el octavo miembro es representante de una consultoría de planificación de transporte. Estamos, por tanto, ante un Consejo formado por cinco académicos, dos cargos públicos y un representante del mundo de la Consultoría. Un consejo formado así con el ánimo de incorporar conocimiento a la gestión ambiental del ejecutivo autonómico, como se puede deducir tanto de su composición como de la metodología empleada.

\section{c) Los foros social y empresarial}

Mediante esta dinámica participativa, la intención del Gobierno Vasco era construir un debate sobre el diagnóstico del medio ambiente en la CAPV y sobre posibles objetivos y actuaciones propuestas desde diferentes ámbitos de actividad. En este sentido, parece que la propuesta llevada a los foros estaba más enfocada en el binomio problemas-soluciones que la de los Desayunos por la Sostenibilidad. En cualquier caso, su desarrollo tuvo lugar a lo largo de un ciclo temporal más amplio, en concreto se desarrollaron cuatro foros en cuatro momentos diferentes, desde marzo hasta noviembre de 2006, teniendo cada uno de ellos una duración de una jornada y estructurándose en distintos grupos de trabajo.

Si atendemos a su composición, participan en los foros un total de ochenta y cinco agentes. Nuevamente, como ocurría en el caso de los Desayunos por la Sostenibili- 
dad, el peso del sector empresarial es más acusado que el de cualquiera de los otros: asociaciones, sector investigador o entidades públicas y semipúblicas. Un total de treinta y cinco agentes económicos acudieron a los foros entre los que se contaban empresas concretas como Eroski S. Coop., Gamesa, Petronor, etc.; numerosos clústeres empresariales, entre ellos ACLIMA, automoción, electrodomésticos, aeronáutica, etc.; organizaciones empresariales: CONFEBASK, Guitrans, Asociación Española de Máquina-Herramienta, etc. y cámaras de comercio así como fundaciones relacionadas con la calidad (Euskalit). En el grupo de las asociaciones encontramos veintiún actores entre los que están los principales sindicatos de la CAPV (incluido el sindicato agrario Euskal Herriko Nekazarien Elkartasuna (Federación de Sindicatos Agrarios de Euskal Herria, EHNE); grupos ecologistas como Eguzki, Ekologistak Martxan o Greenpeace; organizaciones de temática ambiental: Kresala, Federación Vasca de Montaña, etc.; asociaciones de defensa de los intereses de la juventud (Novia Salcedo) o incluso federaciones deportivas (Federación Vasca de Surf). Completaban los foros entidades de investigación, es el caso de las facultades de la UPV, la corporación tecnológica Tecnalia, IK-4, Eusko Ikaskuntza, etc., y entidades públicas o semipúblicas como Radio Euskadi, algunos ayuntamientos, la Asociación vasca de agencias de desarrollo, etc.

\section{GRÁFICO 3}

Proceso participativo en la elaboración del II PMA

(Iniciativa "foros social y empresarial")

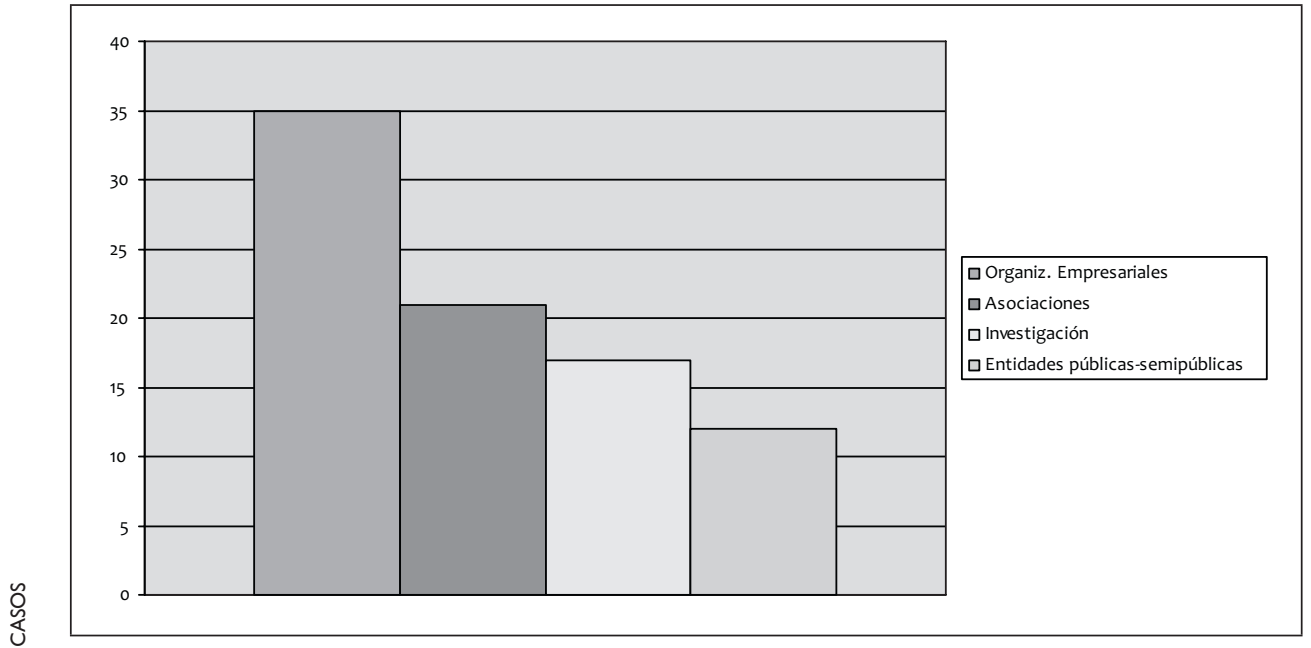

Fuente: Elaboración propia a partir de Gobierno Vasco (2006: 77-78).

Lo cierto es que de esta dinámica participativa no se exponen resultados demasiado concretos en el Anexo 2 del II Programa Marco Ambiental 2007-2010 (Gobierno Vasco, 2006). Se hace hincapié, como no podía ser menos, en las propuestas divergentes que surgen de los foros y prácticamente se hace inventario de las cuestiones tratadas, sin ir más lejos en el carácter de las posturas enfrentadas. La impresión ge-

136 neral es que en este foro se da una cierta neutralización de las opiniones y que no se 
da el paso de operativizar las diferentes propuestas en medidas concretas de actuación. Su relevancia probablemente haya sido mayor a la hora de la divulgación, en tanto la celebración de los foros procura cierto eco social de cara a una mayor sensibilización ambiental de la ciudadanía y un mayor conocimiento por parte de ésta de las actuaciones del ejecutivo autonómico. En todo caso, y más allá de su impacto real, uno de sus organizadores se muestra crítico con los aspectos formales del proceso participativo y el final dado al mismo:

“(... fue un proceso muy centrado en Bilbao, que es lo que pasa muchas veces porque es donde está la mayor parte de la gente...

Otro aspecto (a mejorar) sería... ir configurando esa red también para que la gente no crea que viene para un acto aislado; y que vea que le vas a tener en cuenta, que le vas a informar $y$, por supuesto, que las opiniones que den se van a tener en cuenta y que, además, yo creo que se tienen en cuenta.

(...) el Departamento no supo después mimar de la manera adecuada a la gente que hizo un esfuerzo pues por los Desayunos, o con las entrevistas... sí que hubo un perfil un poco bajo a la hora de cerrar el proceso". (Entrevista $N^{\circ} 13$. Consultor de Medio Ambiente).

Probablemente buena parte de las críticas vertidas por los grupos de la sociedad civil tengan algo que ver con el hecho de que no se aprecia el papel que juega la propia participación, y el esfuerzo de llevarla a cabo, y con la escasa idea de formar parte de un colectivo más amplio ligado de una manera más estable frente a meras acciones puntuales dedicadas al diseño y elaboración de las políticas ambientales.

\section{LA RED DE LA POLÍTICA AMBIENTAL VASCA}

\section{Entramado}

La comunidad de actores en la política ambiental vasca se compone de un elenco que básicamente, como hemos apuntado en la descripción del proceso participativo, podríamos agrupar en cuatro categorías. En primer lugar estarían los actores institucionales entre los que distinguiríamos fundamentalmente a la administración ambiental del resto de administraciones sectoriales, siguiendo la traza del análisis desarrollado por Font (1999: 56-64) en torno a los actores de la política ambiental española. La razón de esta distinción la encontraríamos en una cierta lógica de actuación que ha hecho de la primera, en general, un departamento gubernamental en el que han primado en mayor medida los valores conservacionistas sobre los desarrollistas ${ }^{9}$.

9 Uno de los momentos en los que se evidencia este contraste en el caso vasco se da cuando el por entonces Consejero de Medio ambiente y Ordenación del Territorio, Sabin Intxaurraga, se pronuncia públicamente en contra de algunos proyectos emblemáticos de infraestructuras sobre los que el resto del Ejecutivo, y la gran mayoría de fuerzas políticas presentes y no presentes en el mismo, habían hecho causa común. En diversos artículos publicados en 2004, Intxaurraga criticó abiertamente la construcción de la 
El segundo grupo de actores que componen esta red serían las organizaciones empresariales que han tenido una presencia muy marcada en todo el proceso de diseño, implementación y evaluación de las políticas ambientales, lo que sin duda viene determinado por el hecho de que la política ambiental sea un tipo de política eminentemente regulativo, con costes concentrados en sectores muy concretos y beneficios difusos, asumibles por toda la población. Habría que destacar, sin embargo, como nota general, que las empresas con mayor protagonismo en las políticas ambientales han sido fundamentalmente la propia organización patronal CONFEBASK y las representadas en el clúster ACLIMA. Al respecto se señala:

"Ese es uno de los temas que el gobierno durante los últimos años ha realizado. Es decir, las leyes de medio ambiente y los decretos correspondientes antes de adaptarlos se pasaban a sociedades como por ejemplo a ACLIMA, a CONFEBASK... para que cada una hiciera sus comentarios y muchos de ellos se han tenido en cuenta. O sea, que esa política de participación real entre el Gobierno y la sociedad -y por ésta no me refiero solamente a los individuos o colectivos sociales, sino a las agrupaciones empresariales y demás- ha existido totalmente". (Entrevista $N^{\circ} 6$. Ex alto cargo de Medio Ambiente del Gobierno Vasco).

El mundo empresarial e industrial está en ese entramado, y sobre todo a través del clúster de medio ambiente en el que están los siderúrgicos, los cementeros... y ahí esa élite... pues tienen una influencia muy fuerte". (Entrevista $\mathrm{N}^{\circ} 12$. Ex alto cargo de Medio Ambiente de diferentes administraciones).

No obstante, y según la percepción de algunos informantes, en ese entramado faltaría la representación directa de las empresas más potentes del panorama vasco que optarían por hacerse presentes en otros niveles administrativos más elevados, donde se dirime la normativa básica, o hipotéticamente en las relaciones con otras administraciones sectoriales que presenten líneas de actuación más acordes con su función corporativa:

"A ver, si te voy a ser sincero, por ejemplo creo que las grandes empresas de este país, desde Mondragón hasta la que sea, pasan bastante, al menos, de la política ambiental de la CAPV. Igual pican más alto y tratan de influenciar en la política del Ministerio que tiene una jerarquía más alta, pero yo creo que para las grandes empresas (BBVA, Iberdrola y esas) la política ambiental que se hace en el departamento les queda un poco pequeña. Ellos se preocupan mucho, es mi percepción, de otras cosas. Su guerra está en otro lado... como en el Programa Marco Ambiental que había un Foro empresarial con unas 125-150 personas, pero estas empresas no vienen". (Entrevista № 13. Consultor de Medio Ambiente).

variante sur metropolitana de Bilbao y del Superpuerto de Pasaia ("Dudas sobre la Supersur", El Correo (edición Bizkaia), 29 de julio de 2004: 10 y “Pasaia: ¿es necesario el puerto exterior?”. En línea: <http:// www.nodo50.org/jaizkibel/?p=575> [consulta: 9 enero 2012]), utilizando precisamente el discurso de la sostenibilidad. En los siguientes días quedó claro, a través de las manifestaciones de los integrantes del Gobierno, el Lehendakari a la cabeza, que esta era una postura claramente minoritaria en el gabinete e incluso en su propio partido ( $E /$ Correo (edición Bizkaia), “Ibarretxe desautoriza a Intxaurraga y dice que la Supersur es prioritaria para Euskadi”, 27 de julio de 2004:2 - 3). 
Además, para caracterizar con más precisión las pautas de actuación de los grupos empresariales, podríamos distinguir entre lo que denominaríamos planificación estratégica de la política ambiental -la EAVDS, los PMA, etc.- de un ámbito de actividad mucho más concreto como es la elaboración de decretos de desarrollo de normativa y transposición de directivas europeas. En el primero de esos ámbitos, los interlocutores para la autoridad ambiental han sido las organizaciones antes mencionadas a través de mecanismos más o menos formalizados de participación. Por el contrario, la elaboración de decretos o la negociación de acuerdos voluntarios ha sido en mayor medida una cuestión de relación bilateral entre la sociedad pública de gestión ambiental, IHOBE, adscrita al departamento de medio ambiente, y empresas concretas o clústeres sectoriales distintos a ACLIMA. En estas negociaciones la pauta ha venido marcada predominantemente por contactos informales en los que representantes de la empresa o del sector de actividad negocian con técnicos de la autoridad ambiental, aportando conocimiento especializado. Todo esto lo recoge muy claramente uno de nuestros informantes proveniente del sector productivo del acero:

"Nosotros, con la administración, el tipo de contactos que hemos tenido es para resolver temas muy puntuales del sector... para temas muy concretos hasta para llegar a acuerdos voluntarios para los que establecimos unos contactos para ver qué líneas podíamos trabajar.

(... ) Y si son temas más concretos, el trato es más con los técnicos. Por ejemplo, cuando estuvimos haciendo una revisión del Acuerdo Voluntario estábamos en contacto con los técnicos". (Entrevista № 19. Miembro del clúster Acero y Medioambiente).

Por tanto, existe un grupo de actores empresariales especialmente relevante en los procesos participativos para el desarrollo de normativa y programas estratégicos y otro colectivo diferente -compuesto por empresas aisladas o, normalmente, clústeres de concretos ámbitos productivos- que se activa a la hora de negociar aplicaciones normativas que requieren frecuentemente un conocimiento profundo del sector en aras a su aplicabilidad futura. Las negociaciones son aquí más técnicas y menos políticas, pero sin duda han ocupado una parte importante de la actividad del departamento de medioambiente, como por ejemplo a la hora de implantar la Directiva IPPC o los acuerdos voluntarios.

Un tercer grupo de actores vendría compuesto por las organizaciones de la sociedad civil. Evidentemente, éstas son asociaciones voluntarias que persiguen objetivos públicos y hacen mayor uso de incentivos colectivos -identidad, ideología, etc.- para su funcionamiento interno. Tener presente esto es importante por cuanto, aunque hay dentro de este grupo organizaciones más profesionalizadas y estructuradas como las sindicales, en general las asociaciones encuentran serias dificultades para competir con centros tecnológicos y empresas, nutridos de personal altamente cualificado y fuertemente incentivado a nivel económico, en un ámbito como el de la política ambiental que requiere un notable conocimiento técnico. Especialmente, ese es el caso de las organizaciones ecologistas, cuyo principal recurso 
es la buena imagen social de la que disfrutan ${ }^{10}$, pero que encuentran serias dificultades para captar militancia, renovar la misma y dedicar recursos humanos al seguimiento de iniciativas políticas, expedientes administrativos, procedimientos de alegaciones, etc. Aún así, las organizaciones ecologistas y las sindicales han jugado un papel especialmente activo en los cauces formalizados de participación, estableciendo a veces cierta alianza estratégica con profesionales provenientes del ámbito del conocimiento, especialmente la Universidad ${ }^{11}$, como se señala en una de nuestras entrevistas:

"Luego yo creo que en la experiencia de estos grupos para el Programa Marco, lo que yo he visto ahí, es que los sectores más dinámicos son gente vinculada a la universidad, a movimientos asociativos, a ONGs, gente de profesiones liberales, sociedad civil...". (Entrevista N 13 . Consultor de Medio Ambiente).

En cualquier caso, y frente a ese dinamismo -frecuentemente producto de la alta motivación de un número relativamente reducido de personas- el problema principal de las organizaciones ecologistas es su escaso número de militantes. Precisamente, por la naturaleza del objetivo perseguido -"beneficios difusos"- y por la relativamente baja relevancia del problema medioambiental dentro de las preocupaciones de la ciudadanía vasca ${ }^{12}$, las organizaciones ecologistas muestran una cierta debilidad de cara a la movilización de la ciudadanía. Solamente en conflictos puntuales a lo largo de la historia y en casos donde el conflicto ambiental ha derivado en una cuestión más cercana a la mera distribución de recursos, los intereses conservacionistas han derivado en movimientos sociales fuertes, si bien, en este último supuesto, con un discurso menos ideológico y más de tipo Not-in-my-Backyard (NIMBY). En general, diríamos que los NIMBY medioambientales prestan en la mayoría de las ocasiones el músculo movilizador al movimiento ecologista.

Por último, el cuarto grupo lo conformarían los actores relacionados con la investigación y el conocimiento. Aquí incluimos a personas individuales y a agentes colectivos como determinados centros tecnológicos, consultorías, fundaciones, entidades de promoción científica y grupos universitarios de investigación. Todos estos, y algunos otros semejantes, han jugado un papel en este entramado de agentes de la

10 Véase al efecto los datos del Ecobarómetro Social (2008: 21), en los que el 44\% de los encuestados señala a las asociaciones de protección del medioambiente como las que más confianza les genera en temas relacionados con el entorno. El siguiente agente en términos de confianza serían los científicos -con un $16 \%$ - situándose a la cola las empresas que concitan la confianza del $2 \%$.

11 Existe una ingente cantidad de literatura que conecta, a nivel de valores y de participación, a los colectivos profesionales del sector del conocimiento, y externos al mercado, con los movimientos sociales y, en general, las denominadas "nuevas políticas". Es la línea seguida, por ejemplo, por numerosos estudios elaborados utilizando el marco teórico de Inglehart $(1991,1998)$ y su argumento sobre el cambio de valores y el postmaterialismo.

12 A este efecto, puede consultarse las series del Euskobarometro en <http://www.ehu.es/euskobarometro> o los diferentes Sociómetros emitidos por el Gabinete de Prospección Sociológica del Gobierno Vasco, en <http://www1.euskadi.net/estudios_sociologicos/sociometros_c.apl>, donde, por término medio, las cuestiones medioambientales son citadas por un $1 \%$ de los encuestados al posicionarse sobre sus principales preocupaciones. 
política ambiental, principalmente desde su función de reflexión teórica e investigación. Al respecto se señala:

“(... ) (la situación de) la universidad y los centros es muy diferente. Los centros tecnológicos tienen que vivir en gran medida de esto... y los que viven de esto adoptan una posición muy respetuosa... ¿Cómo influyen? ... pues a través de los trabajos que hacen, evidentemente, pero no los veo como un lobby". (Entrevista $\mathrm{N}^{\circ}$ 12. Ex alto cargo de Medio Ambiente de diferentes administraciones).

Por tanto, el principal capital con el que cuentan es el conocimiento, pero no todos ellos lo gestionan de igual manera. En principio, y como advierten nuestro informante, los centros tecnológicos y consultorías son fundamentalmente gestores de recursos. Las políticas medioambientales han supuesto, entre otras cosas, la movilización de unos recursos -por ejemplo, a través de financiación de proyectos de investigación- que en parte han sido gestionados por entidades de este carácter; en ese contexto, la influencia de éstos ha sido más bien la que se ejerce indirectamente a la hora de emitir informes y producir datos. El caso de la Universidad -y de Eusko Ikaskuntza13, podríamos añadir-, por ser una entidad más ajena a los avatares del mercado, se supone que ha ejercido un papel más en línea con la reflexión teórica y crítica que el de consultoras y centros tecnológicos. En todo caso, tenemos que ser conscientes que un número de personas está presentes en varias entidades diferentes, por lo que en la práctica dilucidar quién ejerce qué tipo de influencia no es una cuestión fácil. Y, si ese cierto tránsito de personas se da entre la Universidad y las consultoras, deberíamos decir lo propio entre la industria y determinados think tanks.

\section{Las relaciones entre los actores}

Entrando a analizar la naturaleza de las relaciones entre los agentes descritos en esas cuatro categorías, deberíamos hablar de una relación de intercambio fundamental entre agentes empresariales e investigadores, por un lado, y la Consejería de Medio Ambiente, por otro. Unos y otros formarían una advocacy coalition (Sabatier y Jenkins-Smith, 1999) que, a nuestro parecer, representaría la principal fuerza dinámica en la gestión política del medio ambiente. Por un lado, la alianza entre un sector de la industria, como son las empresas de gestión ambiental, y un sector mayoritario de las consultoras, centros de investigación y Universidad genera conocimiento experto fuertemente demandado por las instituciones en una política altamente técnica. Por otro lado, la autoridad ambiental ofrece a esta alianza de actores outputs normativos y recursos económicos.

En este contexto, y dentro de la red de la política ambiental, encontraríamos otra coalición promotora: la que han establecido las asociaciones ecologistas -princi-

13 Fundamentalmente, a través de un grupo de personas que han venido llevando a cabo un proyecto sobre Desarrollo Sostenible, con iniciativas tales como la celebración en 2005 de un Congreso, grupos de reflexión sobre el concepto de sostenibilidad, cursos formativos relacionados. Véase en <http:// www.eusko-ikaskuntza.org/eu/proyectos/ds/>. 
palmente Ekologistak Martxan y Eguzki- con determinadas fuerzas sindicales más activas en el tema ambiental -CCOO, Langile Abertzaleen Batzordeak (Comisiones de Obreros Patriotas, LAB) y EHNE-. Esta alianza de actores se plantea como contrapunto a la relación simbiótica entre instituciones y agentes económicos y del conocimiento. Existe también intercambio de recursos entre estas organizaciones y las instituciones ambientales -éstas ofrecen recursos económicos, vía subvenciones, e información y aquéllas ofrecen dosis de legitimidad a través de su participación en los mecanismos formales dispuestos- pero sus planteamientos -las ideas que justifican hablar de una advocacy coalition- permanecen más claramente enfrentados. Si los de las instituciones, con sus aliados, podríamos situarlos en los de la modernización ecológica (Carter, 2007: 227-233), los de las organizaciones ecologistas, sobre todo, caen en el ecologismo social (Ibarra y de la Peña, 2006: 152). Unos apuestan por el papel del mercado y la técnica como factor de armonización entre economía y medio ambiente y otros por una transformación profunda de valores, sistema económico y organización social.

Como factor condicionante externo al funcionamiento de esta red, podríamos citar la interrelación entre las administraciones sectoriales y las grandes empresas cuya ausencia de las políticas ambientales mencionábamos anteriormente. Esta idea básicamente es la que queda expuesta en la Figura 1:

FIGURA 1

La red de la política ambiental en la CAPV

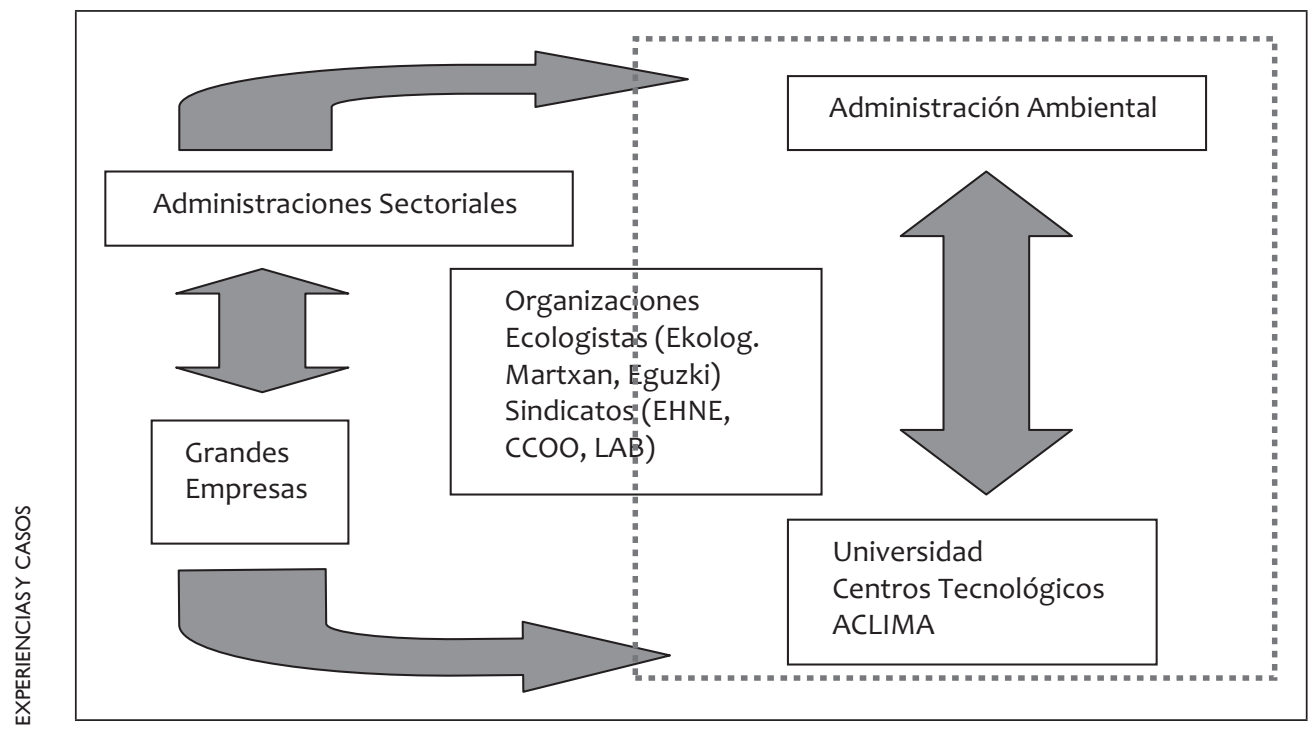

Fuente: Elaboración propia.

De acuerdo a este planteamiento, departamentos de mayor presupuesto y rele-

142 vancia política como transportes o industria, en una relación en la que mantienen vías 
de contacto con empresas y sectores industriales de gran peso específico -empresas energéticas, sectores como la cantería, la automoción, el papelero, etc.- determinan políticas públicas con evidente incidencia ambiental. Estas políticas entran como input en la red de la política ambiental vasca, condicionando tanto el cumplimiento efectivo de la planificación estratégica como la legitimidad de los actores institucionales a ojos de las organizaciones más críticas. En cualquier caso, ese efecto de las administraciones sectoriales va más por el lado de delimitar el margen posible de acción y el impacto real de las políticas ambientales.

En el escenario de la política ambiental, propiamente dicha, la simbiosis entre instituciones y el binomio formado por empresas medioambientales y el sector de la investigación son mutuamente legitimadores. El papel jugado por los actores empresariales se articula a través de sus organizaciones representativas -en especial, ACLIMA- y tiene como colofón la actual apuesta actual de consolidar a la CAPV como polo de eco-innovación (IHOBE, 2009). Como vimos anteriormente, las grandes empresas se ausentan y las de pequeño y mediano tamaño, que no forman parte del mercado de la gestión ambiental y que quizá podrían plantear una mayor conflictividad por tener que cargar con costes suplementarios de producción, asumen como un dato externo las regulaciones ambientales generalmente sin tener una parte activa, como hemos visto, en los mecanismos formales de participación.

En definitiva, y tal y como los apuntan Gomà y Subirats (1998: 382), las políticas ambientales se caracterizan por la débil relevancia política y social de sus contenidos, su elaboración en un contexto multinivel y de complejidad decisional, y su enfoque predominantemente regulativo. Consecuentemente, las redes de actores se caracterizan por una notable impermeabilidad, dándose en ellas una relación dialéctica fundamental: Administración e Industria establecen un sistema de intercambio de recursos económicos, normativos y técnico-cognitivos que deja un espacio restringido a otras organizaciones -especialmente las ambientalistas- que han encontrado pocas oportunidades en los mecanismos de participación. Con las salvedades y matices que pueda presentar el caso vasco, en lo sustancial hacemos propio este diagnóstico para caracterizar la red de la política ambiental en la CAPV.

\section{CONCLUSIONES}

La configuración de la red de política ambiental en la CAPV viene fuertemente condicionada por el carácter de las políticas medioambientales como políticas públicas de costes concentrados y beneficios difusos. Los que reivindican estos últimos han quedado, en el periodo analizado, en una situación de relativa debilidad. Los beneficios de la preservación del medio ambiente son tan difusos y, al mismo tiempo, tan relegados en la escala de valores de la ciudadanía que han determinado una situación de debilidad para las asociaciones ecologistas, en teoría, los valedores más convencidos de esos bienes comunes. Su principal recurso ha venido dado por un cierto capital de legitimidad por su buena imagen que, sin embargo, no se ha traducido en 
militancia. En ese contexto, las instituciones les han reservado un papel en el canal participativo más institucionalizado: el CAMA y los procesos participativos de los programas-marco. Sin embargo, tenemos todos los indicios para pensar que esta participación se ha ceñido exclusivamente al aspecto consultivo de la participación, probablemente sin oportunidades reales de incidir ni en el enfoque, ni en el contenido, ni en los instrumentos diseñados.

En el caso de aquéllos que han tenido que soportar los costes de la política, la historia ha sido diferente. Casi por definición, los intereses empresariales han sido habitualmente intereses fuertes y eficazmente organizados. En este sentido, en la política ambiental han tenido más peso los intereses de los pocos, pero fuertemente afectados, que de los muchos en grado difuso, lo que no es sino una vieja historia en los procesos políticos. Sin embargo, lo que es más relevante es que han sido especialmente aquéllos que han convertido los costes en beneficios -beneficios concentrados- los que se han establecido de manera más sólida como interlocutores de la autoridad ambiental. Nos referimos a esa parte de la industria vasca que en las últimas décadas se ha reciclado para hacer de la producción limpia su nicho de mercado, y que se ha organizado en torno a su propio clúster. Este actor ha tenido fundamentalmente el recurso del conocimiento experto, fuertemente demandado en una política altamente técnica como la medioambiental, y asumida por un departamento gubernamental de recursos limitados. No sorprende, por tanto, que quienes han controlado en mayor medida este recurso -ACLIMA, centros tecnológicos y Universidad- hayan tenido una mayor incidencia en las decisiones en torno a la política ambiental: especialmente relevante en el caso de centros tecnológicos y Universidad para la cuestión del diagnóstico -como se ve en el proceso participativo del II PMA-y más acusado para ACLIMA, en el momento de elegir los instrumentos de actuación. Como hemos visto, en este momento de elegir también se suman, según la ocasión, actores empresariales de los sectores afectados, pero, en cualquier caso, los actores empresariales tienden a involucrarse en mucha menor medida en el CAMA puesto que en su perspectiva parece que el momento efectivo de participación, la posibilidad real de incidir, ha pasado ya cuando este foro se reúne. Por tanto, el paradigma participativo que se asienta con la Ley 3/1998 parece traducirse en un modelo que en gran parte ritualiza la participación, ejerciendo un cierto efecto en la relegitimación de las políticas públicas; circunscribiéndolo a los aspectos consultivos y de diagnóstico. Sin embargo, ese modelo no parece ofrecer un espacio relevante a los grupos de interés público en la toma de decisiones puesto que la participación en un sentido más incisivo, de influir realmente en dicha toma, se reserva a otro tipo de actores -fundamentalmente los empresarialesmediante cauces menos formales de interacción, actores que disponen con mayor profusión del recurso clave: conocimiento técnico, utilizado, por ejemplo, para hacer efectiva la transposición de la normativa comunitaria.

Aún así, ese cambio hacia un modelo más participativo y corresponsabilizador reconfiguró y consolidó efectivamente la red de actores en política ambiental en la CAPV, que parece responder así a una causalidad institucionalista al asumir la norma144 tiva vasca los planteamientos europeos en política ambiental. Los factores institucio- 
nales parecen estar, asimismo, en la raíz de otro argumento que pudiera darse para explicar la evolución de la red: ésta se configura de la manera que lo hace dando centralidad a los actores empresariales, porque se habían sucedido para entonces cambios en el tejido productivo de la CAPV que posibilitaron el surgimiento de un sector industrial medio ambiental. Desde nuestra óptica, también este proceso es una consecuencia de cambios institucionales a nivel europeo que motivan una readaptación en una industria vasca sometida a un proceso de reconversión. En cualquier caso, la importancia de caracterizar la red de la política ambiental va más allá de consolidar explicaciones institucionalistas. La estructura final de la red nos ayuda a explicar mejor el contenido sustantivo de las políticas. En el caso de la política ambiental, por ejemplo, a entender por qué se apuesta más decididamente por un tipo de soluciones que por otras, por qué determinadas políticas subsectoriales son priorizadas y qué tipo de referentes internacionales se buscan para hacer benchmarking. Pero no sólo eso, aparte de las cuestiones sustantivas, la red tiene la virtud de consolidar un enfoque determinado -el de la modernización ecológica en el caso de la política ambiental vasca- que ejerce una influencia decisiva en ulteriores decisiones públicas. Es así como podemos concluir que caracterizar la policy network aporta mayor visibilidad a los acontecimientos presentes y, de no intermediar cambios sistémicos, a las tendencias futuras.

\section{REFERENCIAS BIBLIOGRÁFICAS Y DOCUMENTALES}

Aguilar, S. (1997), El reto del medio ambiente. Conflictos e intereses en la política medioambiental europea. Madrid: Alianza Editorial.

Aguilar, S. (1998), "Las políticas de medioambiente, entre la complejidad técnica y la relevancia social”, en R. Gomà y J. Subirats, coords., Políticas públicas en España. Barcelona: Ariel.

Aguilar, S. (1999), "Supranacionalización y descentralización: nuevos escenarios de la política medioambiental”, en S. Aguilar, N. Font y J. Subirats, eds., Política ambiental en España. Subsidiariedad y desarrollo sostenible. Valencia: Tirant lo Blanch.

Aguilar, S. (2003a), “Hacia el desarrollo sostenible. Evolución y tendencias de la política europea de medio ambiente", Revista Internacional de Sociología, 35: 53-80.

Arenilla, M. (2011), "Redes de políticas: el caso de la ciudad de Madrid", Revista Española de Ciencia Política, 25: 31-56.

Arias, M. y Valencia, A. (2004), “Claves de la emergente política medioambiental española: sostenibilidad, gobernanza y europeización”, Gestión y Análisis de Políticas Públicas, 28-29: 23-39.

Arluzea, E. (2006), "Marco constitucional del medioambiente en la Comunidades Autónomas. La perspectiva vasca", Revista Vasca de Administración Pública, 74: 1150.

Börzel, T.A. (1997), “¿Qué tiene de especial el concepto de policy networks?”, Revista Hispana para el Análisis de las Redes Sociales. En línea: <http://revista-redes.rediris.es/webredes/textos/policynet.pdf> (consulta: 11 julio 2011). 
Carter, N. (2007), The politics of the environment. Ideas, activism, policy. Cambridge: Cambridge University Press.

Cerrillo, A. (2005), La gobernanza hoy: 10 textos de referencia. Madrid: INAP.

Chaqués, L. (2005), Redes de políticas públicas. Madrid, CIS.

De la Peña, A. y Barcena, I. (2012), "Sustainable Development Policies in the Basque Country", en H. Bruyninckx, S. Happaerts y K. Van den Brande, eds., Sustainable Development and Subnational Governments. Policy-making and Multi-level Interactions. Basingstoke, Palgrave Mc-Millan. En prensa.

De la Peña, A., Barcena, I. y Fernandez, J. (2012), Motivos globales, razones europeas. Política medioambiental en la CAPV (1980-2010). Vitoria-Gasteiz: Servicio de Publicaciones del Gobierno Vasco. En prensa.

Font, N. (1999), “Quién es quién en la política ambiental”, en S. Aguilar, N. Font y J. Subirats, eds., Política Ambiental en España. Subsidiariedad y Desarrollo Sostenible. Valencia: Tirant lo Blanch.

Font, N. (2000), “La política medioambiental”, en F. Morata, Políticas públicas en la Unión Europea. Barcelona: Ariel.

Gobierno Vasco (2006), II Programa Marco Ambiental 2007-2010. En línea: <http:// www.ihobe.net/Publicaciones/Ficha.aspx?IdMenu=750e07f4-11a4-40da-840c0590b91bc032\&IdGrupo=PUB\&IdAno $=2007 \& I d T i t u l o=008 \& \operatorname{Cod}=17 f 1 d 398$ egd6-435c-8554-86cd370225a7> (consulta: 11 julio 2011).

Gobierno Vasco (2008), Ecobarómetro Social. Valoración de la población Vasca sobre el Medio Ambiente. Vitoria-Gasteiz: Departamento de Medio ambiente y Ordenación del Territorio.

Gomà, R. y Subirats, J. (1998), Políticas públicas en España: contenido, redes de actores y niveles de gobierno. Barcelona: Ariel.

IHOBE (2009), Euskadi, polo de eco-innovación. En línea: <http://www.ihobe.net/Publicaciones/Ficha.aspx?IdMenu=750e07f4-11a4-40da-840c-0590b91bc032\&Cod= \{1279DC90-BB62-4AB2-81FC-37962AE6EA91\}> (consulta: 11 julio 2011).

Ibarra, P. y De la Peña, A. (2006), "El discurso ecologista de los movimientos sociales”, en A. Valencia, ed., La izquierda verde. Barcelona: Icaria.

Inglehart, R. (1991), El cambio cultural en las sociedades industriales avanzadas. Madrid: CIS.

Inglehart, R. (1998), Modernización y posmodernización. El cambio cultural, económico y político en 43 sociedades. Madrid: CIS.

Klijn, E. (1998), “Policy networks: an overview”, en W.J.M. Kickert y J.F. Koppenjan, eds., Managing complex networks. Londres: Sage.

Morata, F. y Hanf, K. (2000), Gestión pública y redes de políticas públicas: la gobernación del medioambiente en Cataluña. Barcelona: Institut de Ciences Politiques i Socials.

Navarro, C. (1999), “La caracterización de las políticas ambientales de la Unión Europea”, Gestión y Análisis de Políticas Públicas, 15: 95-108.

Natera, A. (2004), “La noción de gobernanza como gestión pública participativa y reticular", Documentos de Trabajo Política y Gestión 2. Madrid: Universidad Carlos III de Madrid. En línea: <http://e-archivo.uc3m.es/bitstream/10016/590/1/ 146 cpa040202.pdf> (consulta: 11 julio 2011). 
Peters, B.G. (2007), “Globalización, gobernanza y Estado: algunas proposiciones acerca del proceso de gobernar", Revista del CLAD Reforma y Democracia, 39: 3350. En línea: <http://www.aoli.com.ar/UNIDAD\%20III/articulo\%20PETERS.pdf> (consulta: 11 julio 2011)

Sabatier, P.A. y Jenkins-Smith, H.C. (1999), "The Advocacy Coalition Framework: An Assessment", en P.A. Sabatier, ed., Theories of the policy process. Boulder: Westview Press. 
